\title{
Repercussão respiratória funcional após colecistectomia com incisão subcostal. Efeito analgésico da morfina
}

\author{
Functional respiratory repercussion in opened subcostal cholecystectomy. \\ Morphine effect analgesic
}

\author{
Gilson Cassem Ramos; Edísio Pereiraz; Salustiano Gabriel Neto - TCBC-GO 3; Enio Chaves de Oliveira - TCBC-GO 4
}

\section{R E S U M O}

\begin{abstract}
Objetivo: Avaliar a função pulmonar pós-colecistectomias subcostais abertas de pacientes sob ação da morfina no pós-operatório imediato. Métodos: Tratou-se de um estudo prospectivo, onde se avaliaram espirometrias pós-operatórias de 15 pacientes submetidas à colecistectomias abertas subcostais, que receberam dose única de morfina peridural na anestesia. Os dados pósoperatórios foram comparados aos pré-operatórios pelo teste t-Student emparelhado. Um valor de $p<0,05$ foi considerado estatisticamente significativo. Resultados: Existiram diferenças significativas para as variáveis Capacidade Vital Forçada $(p=0,007)$ e Volume Expiratório Forçado no $1^{\circ}$ segundo $(\mathrm{p}=0,008)$ no pré e pós-operatório imediato, indicando distúrbios ventilatórios restritivos. Todas as pacientes apresentaram espirometrias normais no $3^{\circ}$ dia de pós-operatório. Conclusão: Mesmo sob ação analgesia da morfina peridural, no pós-operatória imediato, foram observados distúrbios ventilatórios restritivos leves póscolecistectomias subcostais abertas. Contudo, observou-se uma rápida recuperação da função pulmonar, o que pode diminuir a morbidade pulmonar pós-operatória.
\end{abstract}

Descritores: Testes de função respiratória. Período pós-operatório. Colecistectomia/efeitos adversos. Morfina.

\section{INTRODUÇÃO}

$\mathrm{O}$ perações em andar superior de abdome tendem a evoluir no pós-operatório com distúrbios ventilatórios restritivos pós-operatórios ${ }^{1}$. Isto ocorre mais acentuadamente nos procedimentos abertos, contudo são observados também, em menor monta, nos laparoscópicos². A fisiopatogênese destes distúrbios está relacionada principalmente com a inibição reflexa do nervo frênico, induzindo à disfunção diafragmática3,4. Outros fatores, porém, devem ser considerados, dentre eles, o principal é a dor pósoperatória, que é tida como relevante em procedimentos cirúrgicos em andar superior do abdome ${ }^{5,6}$. Assim, autores apontam para menor disfunção ventilatória pós-operatória, inclusive com redução de morbidade ${ }^{7}$, quando a dor pós-operatória é tratada de maneira eficaz. Nesta pesquisa avaliou-se a função pulmonar pós-operatória de pacientes que se submeteram à colecistectomias e receberam perioperatoriamente morfina peridural. A administração da morfina diretamente no canal espinhal é um dos métodos mais eficazes para o tratamento da dor aguda e intensa, como a que ocorre em pós-operatórios. Esse agente se liga aos receptores opióides específicos (mu-1) promovendo analgesia supra-espinhal intensa. Assim, a duração de ação dessa droga no espaço peridural, em dose única, pode perdurar por mais de 24 horas ${ }^{8,9}$, permanecendo o pacien- te sem dor, nesse intervalo de tempo. Isto é, exatamente o momento mais intenso de crise álgica pós-operatória: o pósoperatório imediato. Somente uma fração muito pequena de morfina atinge seu sítio de ação, quando injetada por via parenteral (intramuscular ou endovenosa). Isso se deve à sua baixa lipossolubilidade, motivo pelo qual sua eficácia é bem inferior àquela observada, quando a droga é injetada pela via peridural ${ }^{10}$. Este estudo comparou um grupo de pacientes que se submeteram a colecistectomias subcostais abertas, tratados com morfina peridural na pré-indução anestésica, cuja finalidade foi de promover menores distúrbios ventilatórios e abreviar a recuperação da função pulmonar, que foi avaliada por meio de espirometrias seriadas. Seus objetivos foram, nos pós-operatórios de colecistectomias abertas subcostais, detectar distúrbios ventilatórios restritivos, sua gravidade e o tempo de normalização das espirometrias.

\section{MÉTODOS}

Este estudo foi realizado em pacientes que procuraram o Serviço de Cirurgia do Aparelho Digestivo do Hospital Ortopédico de Goiânia, depois de aprovado pelo Comitê de Ética em Pesquisa do Hospital de Urgências de Goiânia e de ter se obtido o Termo de Consentimento Livre

Trabalho realizado e vinculado ao Programa de Pós-Graduação em Ciências da Saúde da UnB-DF - BR.

1. Título Superior em Anestesiologia e Doutor pela UnB-DF-BR; 2. Professor Doutor do Programa de Pós-Graduação da Faculdade de Ciências da Saúde da UnB - DF-BR; 3. Professor Assistente de Técnica Operatória da Faculdade de Medicina da UFG - GO-BR; 4. Professor Doutor da Disciplina de Coloproctologia da Faculdade de Medicina da UFG - GO-BR. 
e Esclarecido das enfermas envolvidas na pesquisa. Foram escolhidos, aleatoriamente, 15 pacientes do Sistema Único de Saúde (SUS), do gênero feminino, entre 21 e 65 anos, com Índice de Massa Corpórea (IMC) menor ou igual a 35, estado físico ASA (American Society of Anesthesiologists) I ou II, com espirometria, gasometria arterial e radiografia de tórax pré-operatórias normais, e que se submeteriam à colecistectomia sem colangiografia intra-operatória. Excluíram-se as enfermas em uso de fármacos com efeito broncodilatador; tabagistas; pacientes cuja estatura não pode ser determinada com precisão (cifoescoliose, amputação de membros inferiores, acamados, etc.); gestantes; portadoras de doenças respiratórias; abdome agudo ou com história médica pregressa de doença diverticular do cólon; enfermas com antecedentes de úlcera gastro-duodenal; pacientes com antecedentes clínicos de hemorragia digestiva; portadoras de doença neuro-muscular; pacientes psiquiátricas; enfermas com contra-indicação de receberem bloqueio anestésico peridural; e pacientes com atencedentes clínicos de alergia à dipirona, diclofenaco de sódio ou aos anestésicos selecionados para uso. Procedimento, cujo tempo anestésicocirúrgico ultrapassava uma hora, era excluído do estudo e nova paciente era selecionada para compor o grupo.

Tratou-se de um estudo prospectivo, em que as pacientes foram acompanhadas, pós-operatoriamente, avaliando suas funções pulmonares. As enfermas foram submetidas à colecistectomias abertas subcostais, sob bloqueio anestésico peridural com $25 \mathrm{ml}$ bupivacaína $0.375 \%$ mais $0,03 \mathrm{mg} \cdot \mathrm{kg}^{-1}$ de cloridrato de morfina e anestesia geral, com protocolo anestésico padronizado, inclusive com todos os atos anestésicos realizados sempre pelo mesmo anestesiologista. Depois da intubação orotraqueal com colocação de cânulas, número 7,5 e insuflação do balonete com $5 \mathrm{ml}$ de ar, as anestesias foram mantidas com isofluorano (0,5 a 1\%) e $\mathrm{N}_{2} \mathrm{O}$, em uma mistura de $50 \%$ com $\mathrm{O}_{2}$. Dipirona, $2 \mathrm{~g}$, endovenosamente e, diclofenaco de sódio, $75 \mathrm{mg}$, intramuscular, foram administrados, respectivamente, de seis em seis horas e de 12 em 12 horas, iniciando na alta da sala de recuperação pós-anestésica. As pacientes foram operadas sempre pelo mesmo cirurgião, com a mesma técnica cirúrgica ${ }^{11}$. A paciente era posicionada em decúbito dorsal horizontal e submetida à incisão subcostal direita de 5,0 a $7,0 \mathrm{~cm}$, tipo Kocher. Identificavam-se a artéria e ductos císticos, procedendo-se suas ligaduras. Dissecava-se a vesícula biliar de seu leito hepático, procedendo a hemostasia do mesmo. A incisão era fechada por planos: peritônio, aponeurose e pele. As enfermas submeteram-se à espirometrias e gasometrias de sangue arterial seriadas. A primeira espirometria era realizada no pré-operatório. A segunda, no dia seguinte ao procedimento, nas primeiras 24 horas do pós-operatório imediato. A partir deste momento, a cada dois dias, realizaram-se novos exames, até a obtenção de um teste normal para a paciente analisada, momento em que se interrompia a realização de novos exames. As espirometrias foram realizadas sempre pela mesma profissional, Técnica em Função Pulmonar e utilizando-se o mesmo aparelho: espirômetro portátil Spiro Pro ${ }^{\circledR}$ versão 2.0, validado pela American Thoracic Society (ATS), capaz de medir parâmetros pulmonares de fluxo e volume. Esse equipamen- to, além de gerar as curvas de Fluxo/Volume e de Volume/ Tempo, discrimina 12 variáveis espirométricas. Os resultados podem ser impressos, automaticamente, conectando o cabo de impressão do espirômetro em uma impressora. Os parâmetros são analisados baseados na equação de regressão de Knudson ${ }^{12}$. A preparação para cada sessão de espirometria incluía calibrar o espirômetro com seringa apropriada de 31 e ajustá-lo de acordo com a temperatura ambiente $\left(25\right.$ a $\left.40^{\circ} \mathrm{C}\right)$ e pressão atmosférica $(680 \mathrm{mmHg})$. Foram proscritos para as pacientes, nas últimas quatro a seis horas, bebida alcoólica, alimentos que contenham cafeína, tais como chá, café, chocolate e refrigerante tipo coca-cola, pelo seu efeito broncodilatador ${ }^{13}$. As variáveis individuais, estatura (em $\mathrm{cm}$ ), peso (em kg), gênero feminino e data de nascimento, eram coletadas e armazenadas no espirômetro. Após dez minutos de repouso em ambiente calmo, a enferma era orientada a se colocar em posição sentada e a focar sua atenção para a explicação do procedimento, que era descrito, criteriosamente, dando-se ênfase à necessidade de evitar vazamentos em torno da peça bucal descartável e à importância da inspiração máxima seguida de expiração rápida, potente (explosiva) e sustentada, até que se ordenasse sua interrupção. A narina da paciente era obstruída com clipe nasal e o teste realizado em um sistema do tipo fechado. Cada paciente submetia-se a três testes válidos e reprodutíveis. O aparelho Spiro Pro ${ }^{\circledR}$ versão 2.0 utiliza o maior valor obtido da equação Capacidade Vital forçada (CVF) + Volume Expiratório Forçado em 1 segundo $\left(\mathrm{VEF}_{1}\right)$ para selecionar o melhor teste. Os laudos das espirometrias foram fornecidos sempre pelo mesmo pneumologista, especialista em provas de função pulmonar, que as interpretava sem conhecer a história clínica da paciente. A prova broncodilatadora foi incluída na rotina das espirometrias, contudo, os dados obtidos pós-broncodilatação não foram analisados.

As variáveis CVF e VEF, foram analisadas, separadamente, no pré e pós-operatórios, até o momento de suas normalizações (80 \% do valor teórico pré-calculado para $\left(V F\right.$ e $V E F_{1}$ ). A hipótese de igualdade de médias do grupo, antes e após intervenção cirúrgica, foi verificada por meio do teste $t$-Student emparelhado ${ }^{14}$. Um valor de $p<$ 0,05 foi considerado estatisticamente significativo na avaliação de diferenças entre parâmetros.

\section{RESULTADOS}

A tabela 1 refere-se às características demográficas das pacientes e às variáveis operatórias.

Tabela 1 - Características das pacientes estudadas (média \pm desvio padrão).

\begin{tabular}{lrc}
\hline Variáveis & Colecistectomias subcostais \\
\hline Idade(anos) & $41,00 \pm 8,95$ \\
Altura(cm) & $156,40 \pm 4,27$ \\
Peso(kg) & $67,61 \pm 8,29$ \\
IMC & $27,86 \pm 4,22$ \\
\hline
\end{tabular}

$I M C=$ peso $(\mathrm{kg}) /$ altura $\left(\mathrm{m}^{2}\right) ; \min =$ minutos. 
A tabela 2 refere-se às variáveis $\mathrm{CVF}_{\mathrm{V}}$ e $\mathrm{VEF}_{1}$, do pré-operatório ao terceiro dia de pós-operatório. Existiram diferenças estatisticamente significativas para ambas as variáveis, quando se compararam pré $X$ pós-operatório imediato $\left(p=0,0070\right.$ para CVF e $p=0,008$ para $\left.V E F_{1}\right)$ e, para CVF, quando se compararam os valores pré $X 3^{\circ}$ dia de pós-operatório $(p=0,027)$. A tabela 3 refere-se aos valores individuais de cada uma das variáveis espirométricas, no pré e pós-operatórios.

A figura 1 é referente as reduções das variáveis

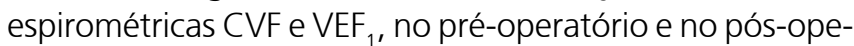
ratório imediato. Já a figura 2 ilustra a curva dessas mesmas variáveis espirométricas, do pré-operatório ao $3^{\circ}$ dia de pós-operatório.

\section{DISCUSSÃO}

Neste estudo verificou-se a presença de distúrbios ventilatórios restritivos leves, mais intensos no pós-ope-

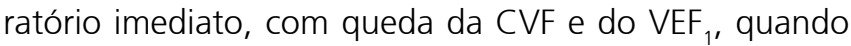
essas duas variáveis são comparadas pré e pós-operatoriamente. Esse achado vem ao encontro de outros, que relatam observações semelhantes, especialmente, no primeiro dia de pós-operatório ${ }^{15}$. A disfunção diafragmática é o fator causal mais importante relacionado aos distúrbios ventilatórios restritivos pós-colecistectomias 3,16,17. Procedimentos cirúrgicos de abdome superior cursam, à radiografia de tórax, com elevação bilateral das cúpulas diafragmáticas e atelectasia em placas ou discóide, com o aparecimento de linhas horizontais ou curvilíneas nos lobos inferiores dos pulmões ${ }^{18}$. Esses achados são observados após operações abdominais e ou na vigência de dor pós-operatória e se relacionam com o enfraquecimento da função diafragmática. A respiração normal em repouso é quase totalmente dependente do movimento do diafragma que, na fase inspiratória, traciona a superfície inferior dos pulmões para baixo ${ }^{19}$, o que faz deste músculo o principal determinante da CVF. Assim, a disfunção diafragmática associa-se com prejuízos dos volumes e capacidades pulmonares, verificados nas espirometrias pós-operatórias de procedimentos cirúrgicos em abdome superior. Outros fatores também são importantes na gênese dos distúrbios ventilatórios, dentre os quais se destaca a dor pós-operatória. A duração do ato anestésico-cirúrgico é causa de morbidade pós-operatória mais significativa, quando ultrapassa de uma a duas horas ${ }^{20,21}$. No presente estudo todo o ato anestésico-cirúrgico, inclusive com extubação traqueal pós-operatória, foi inferior a uma hora. A redução média dos parâmetros CVF e VEF ${ }_{1}$, no pós-operatório imediato, foi, respectivamente, de 19,80 e 20,40\%, em relação aos valores pré-operatórios (Tabela 2 e figura 1). Estas reduções foram inferiores às aferidas em outras pesquisas ${ }^{1,22}$, cujas quedas, para ambas as variáveis, aproximaram de $40 \%$, ou seja, quase o dobro. Pode-se dizer então, que nesse estudo, o efeito analgésico da morfina peridural tende a amenizar a manifestação de distúrbios ventilatórios restritivos pós-colecistectomia aberta, impedindo uma redução pós-operatória da CVF e VEF, mais significativa dessas duas variáveis. Todas as pacientes completaram as normalizações espirométricas, relacionadas aos valores preditos, no terceiro dias de pós-operatório, diferente de outros autores que apontam uma normalização mais prolongada, de sete a dez dias ${ }^{1,23}$. A figura 2 ilustra a evolução das curvas das variáveis CVF e VEF. Embora, no terceiro dia de pós-operatório, o valor médio dessas duas variáveis não tenha alcançado as cifras pré-operatórias, os valores obtidos são considerados normais, relacionados aos preditos calculados pelo espirômetro. Dessa forma, pode-se considerar que a analgesia promovida pela morfina peridural em dose única, na pré-indução da anestesia, parece ser útil, inclusive, para reduzir o período de normalização das

Tabela 3 - Valores individuais (litros) das variáveis espirométricas.

\begin{tabular}{|c|c|c|c|c|c|c|}
\hline \multirow[b]{2}{*}{ Ordem } & \multicolumn{2}{|c|}{ Pré-operatório } & \multicolumn{2}{|c|}{ PO imediato } & \multicolumn{2}{|c|}{$3^{\circ} \mathrm{PO}$} \\
\hline & CVF & $\mathrm{VEF}_{1}$ & CVF & VEF $_{1}$ & CVF & $\mathrm{VEF}_{1}$ \\
\hline 1 & 3,17 & 2,60 & 3,06 & 2,48 & 3,14 & 2,44 \\
\hline 2 & 3,19 & 2,76 & 2,53 & 2,04 & 2,54 & 2,54 \\
\hline 3 & 3,35 & 2,84 & 2,97 & 2,48 & 3,26 & 2,88 \\
\hline 4 & 1,99 & 1,64 & 1,92 & 1,68 & 2,11 & 1,84 \\
\hline 5 & 2,93 & 2,40 & 2,47 & 2,16 & 2,88 & 2,40 \\
\hline 6 & 2,88 & 2,48 & 2,67 & 2,32 & 2,76 & 2,40 \\
\hline 7 & 3,62 & 3,00 & 2,75 & 2,12 & 3,11 & 2,40 \\
\hline 8 & 3,67 & 3,00 & 2,32 & 1,74 & 2,98 & 2,32 \\
\hline 9 & 2,36 & 2,00 & 1,84 & 1,60 & 1,90 & 1,60 \\
\hline 10 & 2,62 & 2,32 & 1,93 & 1,68 & 2,33 & 2,08 \\
\hline 11 & 2,59 & 1,92 & 3,01 & 2,52 & 3,13 & 2,72 \\
\hline 12 & 2,48 & 2,12 & 2,87 & 2,40 & 2,57 & 2,12 \\
\hline 13 & 3,18 & 2,92 & 2,77 & 2,32 & 2,98 & 2,56 \\
\hline 14 & 3,62 & 3,08 & 1,27 & 1,27 & 2,38 & 2,20 \\
\hline 15 & 2,99 & 2,36 & 1,47 & 1,12 & 2,52 & 2,04 \\
\hline
\end{tabular}

PO = Pós-operatório.

Tabela 2 - Variáveis espirométricas: comparação do pré X pós-operatório imediato e pré X $3^{\circ}$ dia pós-operatório (PO).

\begin{tabular}{|c|c|c|c|c|c|c|c|c|c|c|c|}
\hline \multirow[t]{3}{*}{ Variáveis } & \multicolumn{7}{|c|}{ Comparação } & \multicolumn{4}{|c|}{ Estatística } \\
\hline & \multicolumn{2}{|c|}{ Pré-operatório } & \multicolumn{2}{|c|}{ PO imediato } & \multicolumn{3}{|c|}{$3^{\circ}$ Pós-operatório } & \multicolumn{2}{|c|}{$\begin{array}{l}\text { Pré X PO } \\
\text { imediato }\end{array}$} & \multicolumn{2}{|c|}{ Pré $\times 3^{\circ} \mathrm{PO}$} \\
\hline & Média & Desvio Padrão & Média & Desvio Padrão & Média & Desvio & Padrão & $t$ & $p$ & $\mathrm{t}$ & $p$ \\
\hline CVF & 2,98 & 0,50 & 2,39 & 0,57 & 2,70 & &, 41 & 3,131 & 0,007 & 2,475 & 0,027 \\
\hline $\mathrm{VEF}_{1}$ & 2,50 & 0,44 & 1,99 & 0,45 & 2,30 & & , 33 & 3,085 & 0,008 & 1,869 & 0,083 \\
\hline
\end{tabular}




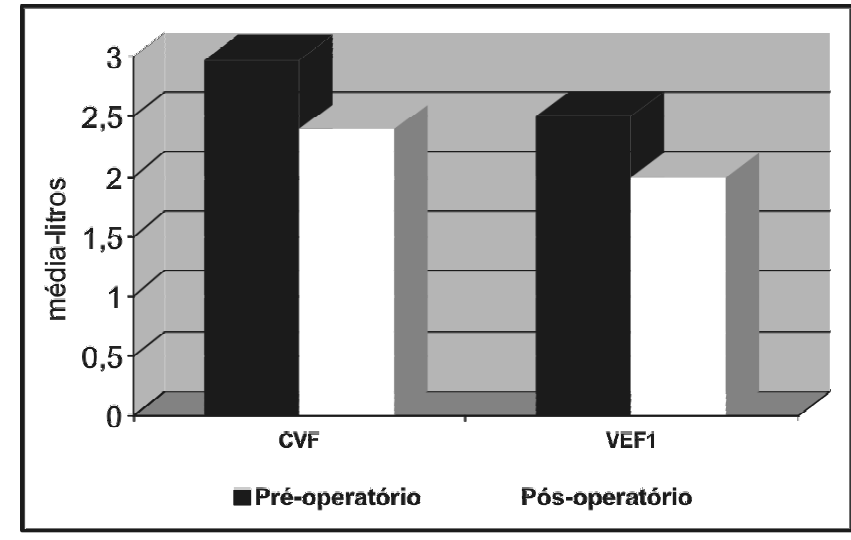

Figura 1 - CVF e VEF : pré-operatório e pós-operatório imediato.

espirometrias pós-operatórias. Mesmo sendo a via laparoscópica a de escolha para colecistectomias, no Brasil se realiza um grande número de procedimentos dessa natureza pela via aberta. Assim, segundo o DataSUS (www.datasus.gov.br), 92\% das colecistectomias realizadas em pacientes do Sistema Único de Saúde (SUS), no ano de 2004, foram pela via aberta. Isso ocorre por uma série de razões, dentre as quais se destacam: falta de recursos para aquisição e manutenção do aparato laparoscópico, grande número de cirurgiões mais velhos que não dominam a técnica mais moderna e aprendizado

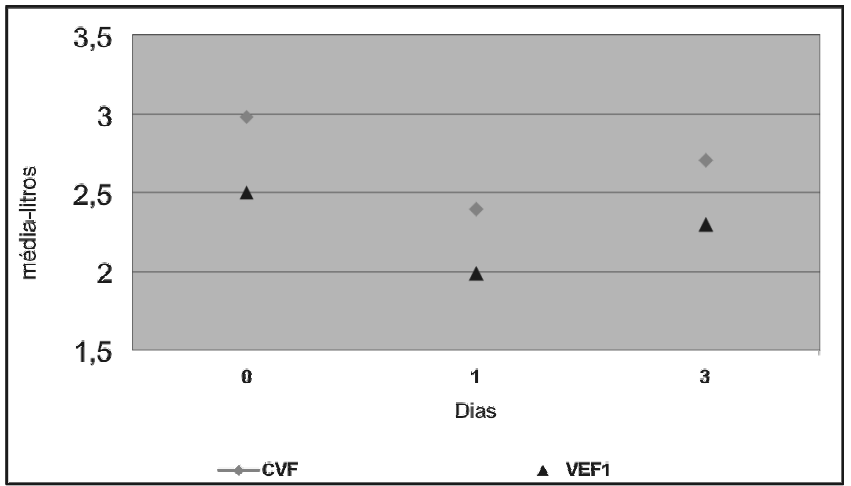

Figura 2 - CVF e VEF : do período pré-operatório até o terceiro dia de pós-operatório.

do cirurgião em especialização. Além disso, existem as contra-indicações relacionadas ao paciente, tais como: colelitíase complicada, doença cardíaca ou cardiorespiratória grave e anormalidades anatômicas de parede ou de cavidade abdominal, causadas, por exemplo, por operações abdominais prévias. Em conclusão, quando se fizer necessário a via aberta para colecistectomia, a morfina peridural, como adjuvante, pode amenizar os distúrbios ventilatórios restritivos pós-operatório, abreviando a normalização espirométrica e, conseqüentemente, reduzir a morbidade pós-operatória.

\title{
A B S T R A C T
}

\begin{abstract}
Objective: To evaluate pulmonary function after open subcostal cholecystecomy under action of the morphine in the immediate post-operative. Methods: This was a prospective study, in which the post-operative spirometries of fifteen patients who underwent open subcostal cholecystectomies which received peridural morphine anesthesia. Post- and pre-operative data were compared using a paired student- $t$ test. A value of $p<0,05$ was considered statistically significant. Results: Significant differences existed for the Forced Vital Capacity variable $(p=0,007)$ and Forced Expiratory Volume in the first second $(p=0,008)$ between pre- and immediate post-operative, indicating restrictive ventilatory disturbances. All of the patients presented normal espirometries in the third day of post-operative. Conclusion: Even under action morphine peridural analgesia, in the immediate post-operative, light restrictive post-cholecystectomy ventilatory disturbances were observed. However, it was observed abbreviated recovery of pulmonary function, which may lower post-operative pulmonary morbidity.
\end{abstract}

Key words: Respiratory function tests. Postoperative period. Cholecystectomy/adverse effects. Morphine. Anesthesia, epidural.

\section{REFERENCIAS}

1. Craig DB. Postoperative recovery of pulmonary function. Anesth Analg. 1981; 60(1):46-52.

2. Erice F, Fox GS, Salib YM, Romano E, Meakins JL, Magder SA. Diaphragmatic function before and after laparoscopic cholecystectomy. Anesthesiology. 1993; 79(5):966-75; discussion 27A-28A.

3. Simonneau G, Vivien A, Sartene R, Kunstlinger F, Samii K, Noviant $Y$, Duroux P. Diaphragm dysfunction induced by upper abdominal surgery. Role of postoperative pain. Am Rev Respir Dis. 1983; 128(5):899-903.

4. Ford GT, Whitelaw WA, Rosenal TW, Cruse PJ, Guenter CA. Diaphragm function after upper abdominal surgery in humans. Am Rev Respir Dis. 1983; 127(4):431-6.

5. McMahon AJ, Russell IT, Ramsay G, Sunderland G, Baxter JN, Anderson JR et al. Laparoscopic and minilaparotomy cholecystectomy: a randomized trial comparing postoperative pain and pulmonary function. Surgery. 1994; 115(5):533-9.
6. Nguyen NT, Lee SL, Goldman C, Fleming N, Arango A, McFall R, Wolfe BM. Comparison of pulmonary function and postoperative pain after laparoscopic versus open gastric bypass: a randomized trial. J Am Coll Surg. 2001; 192(4):469-76; discussion 476-7.

7. Zenz M, Piepenbrock S, Otten B, Otten G, Neuhaus R. [Epidural morphine analgesia. Postoperative period.] Anaesthesist. 1981; 30(2):77-82.

8. Rosen MA, Hughes SC, Shnider SM, Abboud TK, Norton M, Dailey PA, Curtis JD. Epidural morphine for the relief of postoperative pain after cesarean delivery. Anesth Analg. 1983; 62(7):666-72.

9. Fuller JG, McMorland GH, Douglas MJ, Palmer L. Epidural morphine for analgesia after caesarean section: a report of 4880 patients. Can J Anaesth. 1990; 37(6):636-40.

10. Eriksson-Mjöberg M, Svensson JO, Almkvist O, Olund A, Gustafsson LL. Extradural morphine gives better pain relief than patientcontrolled i.v. morphine after hysterectomy. Br J Anaesth. 1997; 78(1):10-6. 
Ramos et al.

11. Ahrendt SA, Pitt HA. Vias biliares. In: Townsend CM, Beauchamp $\mathrm{RD}$, Evers BM, Mattox $\mathrm{KL}$, editores. Sabiston tratado de cirurgia. $17^{a}$ ed. Rio de Janeiro: Elsevier; 2005. p. 1597-641.

12. Knudson RJ, Lebowitz MD, Holberg CJ, Burrows B. Changes in the normal maximal expiratory flow-volume curve with growth and aging. Am Rev Respir Dis. 1983; 127(6):725-34.

13. Sociedade Brasileira de Pneumologia e Tisiologia. I Consenso brasileiro sobre espirometria. J Pneumol. 1996; 22(3):122-9.

14. Vieira S. Teste t. In: Vieira S. Introdução a bioestatística. $3^{a}$ ed. Rio de Janeiro: Campus; 1980. p. 121-36

15. Ramos GC, Pereira E, Gabriel Neto S, Oliveira EC, Rassi RH, Lemos Neto SP. Influência da morfina peridural na funcão pulmonar de pacientes submetidos a colecistectomia aberta. Rev Bras Anestesiol. 2007; 57(4):366-81.

16. Dureuil B, Cantineau JP, Desmonts JM. Effects of upper or lower abdominal surgery on diaphragmatic function. Br J Anaesth. 1987; 59(10):1230-5.

17. Sprung J, Cheng EY, Nimphius N, Hubmayr RD, Rodarte JR, Kampine JP. Diaphragm dysfunction and respiratory insufficiency after upper abdominal surgery. Plucne Bolesti. 1991; 43(1-2):5-12.

18. Barker AF. Bronquiectasia e transtornos parenquimatosos e das vias aéreas localizadas, In: Goldman L, Ausiello D. Cecil. Tratado de medicina interna. 22 ${ }^{\mathrm{a}}$ ed. Rio de Janeiro: Elsevier; 2005. p. 602.

19. Guyton AC. Ventilação pulmonar. In: Guyton AC. Tratado de fisiologia médica. $6^{a}$ ed. Rio de Janeiro: Guanabara; 1986. p. 412.

20. Kroenke K, Lawrence VA, Theroux JF, Tuley MR. Operative risk in patients with severe obstructive pulmonary disease. Arch Intern Med. 1992; 152(5): 967-71
21. Wong $D H$, Weber $E C$, Schell MJ, Wong $A B$, Anderson $C T$, Barker SJ. Factors associated with postoperative pulmonary complications in patients with severe chronic obstructive pulmonary disease. Anesth Analg. 1995; 80(2):276-84.

22. Ravimohan SM, Kaman L, Jindal R, Singh R, Jindal SK. Postoperative pulmonary function in laparoscopic versus open cholecystectomy: prospective, comparative study. Indian J Gastroenterol. 2005; 24(1):6-8

23. Ali J, Weisel RD, Layug AB, Kripke BJ, Hechtman HB. Consequences of postoperative alterations in respiratory mechanics. Am J Surg. $1974 ; 128(3)$ : 376-82.

Recebido em 19/09/2008

Aceito para publicação em 14/11/2008

Conflito de interesse: nenhum

Fonte de financiamente: nenhum

\section{Como citar este artigo:}

Ramos GC, Pereira E, Gabriel Neto S, Oliveira EC. Repercussão respiratória funcional da colecistectomia aberta subcostal. Efeito analgésico da morfina. Rev Col Bras Cir. [periódico na Internet] 2009; 36(2). Disponível em URL: http://www.scielo.br/rcbc

\section{Endereço para correspondência:}

Gilson Cassem Ramos

E-mail: gilson.ramos@terra.com.br 